\title{
Interpreting statistical testing: Process and propensity, not population and random sampling
}

\author{
ROBERT W. FRICK \\ State University of New York, Stony Brook, New York
}

\begin{abstract}
The standard textbook treatment of conventional statistical tests assumes random sampling from a population and interprets the outcome of the statistical testing as being about a population. Problems with this interpretation include that (1) experimenters rarely make any attempt to randomly sample, (2) if random sampling occurred, conventional statistical tests would not precisely describe the population, and (3) experimenters do not use statistical testing to generalize to a population. The assumption of random sampling can be replaced with the assumption that scores were produced by a process. Rejecting the null hypothesis then leads to a conclusion about process, applying to only the subjects in the experiment (e.g., that some difference in the treatment of two groups caused the difference in average scores). This interpretation avoids the problems noted and fits how statistical testing is used in psychology.
\end{abstract}

The calculations of most statistical tests assume that scores are independent, identically distributed random variables (iids). Such tests include the $t$ test, the $F$ test, and Pearson's $r$. The question is, how should this assumption be justified? The traditional justification is to assume that the scores were sampled randomly from a population. This assumption was used by Gosset in his presentation of the $t$ test (Student, 1908), and it can be found in most statistical textbooks in psychology.

The assumption of random sampling from a population has no effect on the statistical calculations, but it determines the interpretation given to the outcome of the statistical test - the null hypothesis is about the populations from which scores were sampled. For example, in a twosample $t$ test, the null hypothesis might be that the scores were sampled from the same population (or two populations with the same mean). The conclusion that follows from rejecting the null hypothesis hence is about the population from which scores were sampled. This will be called the population-based interpretation of statistical testing.

In this article, I argue that the assumption of random sampling from a population is unjustified, unnecessary; inaccurate, and does not serve psychology well. Instead, the treatment of scores as iids can be justified by the process of producing scores. This leads to a process-based interpretation in which statistical testing is used to make claims about the process of producing scores for the particular subjects in the experiment. For example, for the twosample $t$ test, it could lead to the conclusion that an observed difference in group means was not caused by chance

I thank Darla Broberg, David Cross, William Morrison, Howard Sandler, George Wolford, and anonymous reviewers for commenting on previous drafts of this article. Correspondence should be addressed to R. W. Frick, Department of Psychology, State University of New York, Stony Brook, NY 11794-2500 (e-mail: rfrick@psychl.psy.sunysb.edu). fluctuations, but instead by some systematic difference in the treatment of the two groups. This interpretation fits how experimenters use statistical testing.

\section{BASIC PROBLEMS WITH THE NOTION OF RANDOM SAMPLING}

\section{In Search of a Population}

One basic problem with the assumption of random sampling is that if it did not occur, it cannot provide any justification for treating the scores as iids. In practice, experimenters rarely make any effort to randomly sample from a population (Edgington, 1966). Furthermore, they do not identify a population before performing their experiment, and they do not treat the failure to randomly sample as a methodological error. For example, when I perform an experiment, I post a sign-up sheet. Some students from introductory psychology classes at Stony Brook who are looking to sign up for an experiment will choose my experiment over the other experiments posted. Then, most but not all of these students will show up for my experiment and be tested. There is no sign of random sampling in this procedure, I have no intention to randomly sample, and my peers will not criticize my lack of random sampling.

In order to retain the assumption of random sampling yet legitimize the use of statistical testing when random sampling does not occur, people have tried to find or construct a population from which random sampling could be said to have occurred.

Real populations. One possibility is to admit that my population is not all people, but claim that it is some more narrow population of people, such as all college students. However, I tested only students at Stony Brook. If my interest actually was generalizing to all students (e.g., to predict how students would vote in an election), this would be a serious bias and considered inappropriate 
methodology. Therefore, I cannot be said to have randomly sampled from all college students.

One could try to claim that I randomly sampled from students at Stony Brook, but in fact I tested only students who were currently enrolled in introductory psychology. One could try to say that I randomly sampled from students taking an introductory psychology class at Stony Brook, but I tested only students who were signing up for an experiment at a particular time during the semester. Also, I tested only students who were interested in my experimental description and who were responsible enough to show up for the experiment.

One could say that I sampled randomly from all students at Stony Brook taking introductory psychology, who (1) were looking for an experiment at that time, (2) chose my experiment, and (3) showed up the next week. However, my subjects are the only people in the universe fitting this description. Thus, there is no population larger than my sample from which my subjects can be called an unbiased sample.

Furthermore, at some point in this attempt to shrink the population to avoid bias, the population becomes so small and restricted that the generalization to the population is uninteresting - what is the point in generalizing from a narrow sample to a narrow population?

Populations like the sample. A physicist does not need to sample randomly from all electrons to generalize the results to all electrons, because all electrons are the same. Similarly, if a population of people can be identified in such a way that the people in the class are all "alike," any sample from this population will be as good as a random sample and hence functionally equivalent to a random sample.

Unlike electrons, though, no two people are exactly alike. Therefore, there is no larger population that is exactly the same as my subjects, just as my subjects are all different from one another. I could try to identify the typical subject, such as being reliable and 18-20 years old, and then claim that I have randomly sampled from this population. However, some of my subjects will not fit this type. It is illogical to claim that I sampled from a population that does not include all of the subjects in my sample.

One could try to claim that the population is people exactly like the subjects with regard to the effect of the independent variable on the dependent variable. For example, if my experiment is about the effect of a drug on depression, the population could be all people who would show the same effect. However, if the population is known to exactly match the sample, statistical testing would not be needed to generalize from the sample to the population.

Hypothetical populations. Abandoning the search for a real population, many have claimed that random sampling occurred from a hypothetical population of scores. One problem with this is that if the hypothetical population exactly matches the sample, the population can be known exactly-it would be the same as the observed sample--and there would be no need for statistical testing. On the other hand, it is not clear how the hypothetical population could be any different from the sample.

A second problem is that there is no obvious value in generalizing to a hypothetical population. Scientists do not make claims about hypothetical populations, and treating the outcome of statistical testing as being about hypothetical populations leads to logical absurdities. For example, suppose I claim that the $95 \%$ confidence interval for the outcome of a measurement was from 6 to 10 . It is not clear how the true value in the hypothetical population could be outside this range, but if it was, so what? There surely is some other hypothetical population for which this claim is true. More generally, any conclusion is correct for some hypothetical population.

Or, if experimenters can assume sampling from a hypothetical population, why not assume stratified sampling instead? The results of the statistical testing would then be more precise, increasing the power of the experiment and reducing the size of confidence intervals. Of course, it would be difficult to justify that stratified sampling occurred when it did not, but this would seem to be no more difficult than claiming random sampling occurred when it did not.

The underlying problem. Thus, there appears to be no method of post hoc constructing a population from which random sampling could be said to have occurred. The underlying problem is that random sampling is a process, not the outcome of a process. This process has important properties, such as that (1) each item in the population is equally likely to be selected, and (2) the sample tends to differ from the population yet resemble the population (e.g., as described by the law of large numbers). Choosing a population post hoc does not create the process of random sampling; hence it does not create the properties of random sampling.

For example, Kendall (1952) reported trying to imagine how all of the other members of the hypothetical population, even though imaginary, nonetheless had the same possibility of becoming real as the ones that occurred, and that in some way the actual event was "chosen" to occur. Kendall reports being baffled.

When statistical testing is used to make an inference from a sample to a population, the underlying notion is that because of sampling error, the population does not necessarily match the sample. There is no method of choosing a population so that the sample tends to resemble the population as described by the law of large numbers.

Defining the hypothetical population in terms of process. Wallis and Roberts $(1956$, p. 126) attempted to define the hypothetical population in terms of process, as "the totality of numbers that would result from indefinitely many repetitions of the same process of selecting objects, measuring or classifying them, and recording results." I think this definition, based on process, is the best attempt to construct a hypothetical population. It seems 
at first to neatly create a population of scores that might differ from the sample but does not have any systematic differences.

However, the process I used to select my subjects produced only my subjects. To produce more subjects, I would have had to use at least a slightly different process. Any change in my process, particularly one that could yield an infinite number of subjects, would produce a population that was systematically different from my actual sample. For example, leaving up my sign-up sheet for a longer duration produces slightly different subjects, because the nature of the subjects changes over time. Posting my signup sheet at other universities would produce more subjects but again change the nature of my subjects.

\section{Should There Be a Real Population?}

So far, the attempt has been to justify statistical testing in the absence of random sampling by constructing some population from which random sampling occurred. The opposite approach would be to say that experimenters cannot use statistical testing unless they actually use random sampling from a real population and that their research is methodologically flawed when it does not. In the present context, this claim begs the question-it assumes that the only possible justification for thinking of the scores as iids is random sampling from the population, which I will argue is not correct.

But for a moment take this viewpoint seriously. According to this view, I should select a population, such as the students at Stony Brook taking introductory psychology, and then attempt to randomly sample from them. As already noted, it is not clear what this would accomplishwhat great advantage is to be accrued by generalizing the results from my subjects to all of the introductory students that semester?

This procedure would at least satisfy the demands of random sampling. Unfortunately, I cannot coerce the randomly selected subjects into being in my experiment, so I cannot randomly sample from this population. But there is a population from which I could randomly sample. I could have 100 subjects participate in my experiment and then delete the data from 50 of these subjects, chosen randomly. The scores from the remaining 50 would then be a random sample, on which I could perform statistical testing.

Obviously, this procedure is absurd-I am deleting data just to satisfy the assumption of random sampling and then using statistical testing to make inferences about only the deleted data. My conclusion would be more accurate if I simply used the data from all subjects, but of course then I would not have random sampling. The point is, taking the demand for random sampling seriously leads to an absurd solution.

\section{The Results of Statistical Tests Do Not Describe Finite Populations}

A second basic problem with the random sampling interpretation is that the outcome of statistical testing de- scribes the parameters of the iids, not the parameters of the population, and these two are not necessarily the same. In other words, if an investigator actually sampled randomly from a population, the outcome of a conventional statistical test would not exactly describe that population (Godambe, 1955).

For example, suppose that (1) I wanted to know the average grade point average of students at a local high school, (2) I randomly sampled 40 students, and (3) the result was a grade point average of 2.50 with a standard error of .12. Using the $t$ distribution, my $95 \%$ confidence interval would be approximately $2.50 \pm .24$. This confidence interval does not apply to the population of students at this high school, and it obviously does not apply to the students whose grade point average is already known. Instead, the confidence interval for this high school depends on the number of unsampled students. At the extreme, if the school had 41 students, a $95 \%$ confidence interval would be approximately $2.50 \pm .04$ because 40 of the grade point averages are known and the remaining student is $95 \%$ probable to be in the range from 1.00 to 4.00 .

The same discrepancy occurs for statistical testing. Suppose I compared two random samples of 40 students from two different high schools. One school had an average grade-point of 2.70 , the other had an average gradepoint of 2.50, and again there was a standard error of .12 . A $t$ test would reveal $p>.05$ for this difference in grade points. However, if each high school had only 41 students, then the two high schools would have to differ in grade point average - with only 2 unsampled students, the smallest possible difference between these two schools would be .10 . Thus, the true value of $p$ is 0 but the $t$ test yielded $p>.05$.

The practical implications of this discrepancy are minor. When the sample is small relative to the population, the discrepancy in estimation is small and can be ignored $(10 \%$ is the usual cutoff, Hahn \& Meeker, 1991). The philosophical implications, however, are telling - most conventional tests were not designed to make conclusions about real populations.

The statistical test will exactly describe the population if the population is infinite. Therefore, to justify the population-based interpretation, the population could be assumed to always be infinite. However, claiming that generalization occurs to an infinite population sidesteps the underlying problem. The fact remains that at a conceptual level, conventional tests are not trying to describe the parameter of a population.

\section{THE PROCESS-BASED INTERPRETATION}

It has been suggested (e.g., Bradbury, 1987; Edgington, 1987) that because conventional statistical tests rely on the assumption of random sampling and because random sampling does not occur, (1) use of conventional statistical tests is not justified, and (2) other tests, such as randomization or permutation tests, should be used instead. However, there is another justification for conven- 
tional statistical tests that does not rely on the assumption of random sampling.

\section{The Assumption of Process}

Consider the process of flipping a coin. It is reasonable to model the coin flips as being iids. These random variables would take the values of heads or tails, with a constant but perhaps unknown probability of heads. This uses a "propensity" interpretation of probability, discussed in the Appendix, in which the coin is assumed to have some propensity on each trial to produce heads.

Now consider the subjects in a particular condition of an experiment, such as the control group of a simple twogroup design. There is a process leading to an outcomesubjects are tested, yielding a score on some dependent measure. Assuming independence, 1 it is again reasonable to model the scores as being iids. This again assumes a propensity interpretation of probability - subjects have a propensity to produce scores with a given distribution. (In most conventional tests, this is assumed to be a normally distributed random variable with an unknown mean and standard deviation.)

\section{Justifying the Assumption of Process}

The assumption of a process is so general that it must be true - the scores must have been produced by some process. The process should be conceptualized as something that has actually occurred, to legitimize the statistical test. For a between-subjects design, the process could be thought of as the entire life history of the subjects, with the different experiences and genetic history of the subjects contributing to the chance fluctuations. More simply, the process could be conceptualized as being the random assignment of subjects to groups, with differences in individual ability creating chance fluctuations.

\section{The Meaning of the Outcome}

Just as the assumption of random sampling leads to a population-based interpretation, the assumption of process leads to the process-based interpretation - the null hypothesis is phrased in terms of process, so rejecting the null hypothesis leads to a conclusion about process.

For example, suppose a treatment condition is being compared with a control condition, with random assignment of subjects to the two groups. The subjects in each group have a certain propensity to produce scores with a given mean, but this propensity is masked by chance factors. These chance factors would include measurement error and the chance factors associated with random assignment. Thus, in the treatment group, the scores could be modeled as being $\mu_{1}+\varepsilon$, and in the treatment group the scores could be modeled as $\mu_{2}+\varepsilon$. If a difference between groups was observed, the question would be, was this difference caused by the fact that $\mu_{1} \neq \mu_{2}$, which is to say, because the differential treatment of the two groups had an effect? Or did the treatment have no effect- $\mu_{1}=\mu_{2}$ and the difference was caused by chance factors? Rejecting the null hypothesis would allow the conclusion that the difference between groups was not caused by chance fluctuation in the process but instead by some systematic difference in the treatment of the two groups.

To contrast the two interpretations, suppose the observed result of an experiment is a difference between two means in the subjects tested. In the population-based interpretation, statistical significance is used to conclude that the means also differ in a population. Therefore, the inferential jump is from the sample to a population, but the nature of the claim does not change. In contrast, the conclusion from the process-based interpretation is still about the sample. However, the nature of the claim changesthe conclusion is about the reason for the difference between means.

Of course, the statistical test does not address the possibility of confounds. A lack of confounds, coupled with statistical significance, leads to what I call the finding (Frick, 1996). For the true experiment, the finding is a causal claim about the subjects in the experiment. For example, a simple finding is that the manipulation of the independent variable caused changes in the dependent variable.

For the correlational study-for example, determining the correlation between two measures - the finding is a little different. The assumption again would be that scores are produced by some process. The typical null hypothesis would be that the scores have no propensity to be correlated, which is to say, nothing is causing them to be correlated. Rejecting this null hypothesis implies that the two measures have a propensity to be correlated, for the subjects tested. This propensity could arise because changes in the first variable cause changes in the second, changes in the second variable cause changes in the first, or because changes in some third variable cause changes in both of these variables.

\section{Accuracy}

As noted, the outcome of statistical testing does not exactly describe a real population from which random sampling occurred. The outcome of statistical testing instead applies to the parameters of the iids. With the processbased interpretation, the conclusion is about these iids. Therefore, the outcome of statistical testing exactly fits the process-based interpretation.

For example, the confidence interval of $2.50 \pm .24$, based on a random sample of 40 subjects from a high school, applies to the process of producing grades in this high school. The actual number of students in the high school is irrelevant. Similarly, a $t$ test comparing random samples from two schools applies to the process of producing grades at the two schools.

\section{Use of the Process-Based Interpretation}

Statistical writers sometimes use the process-based interpretation in their informal discussions. For example, Fisher (1949) assumed random sampling from a population in his formal descriptions of statistical testing. However, in discussing his experiment of the lady tasting tea 
and claiming that she can know which ingredients were added first, he wrote that the null hypothesis was "that the judgments given are in no way influenced by the order in which the ingredients have been added" (pp. 15-16). This is a claim about process, not populations.

The process-based interpretation also fits how experimenters use statistical testing. Consider the well-known schema for experimenting proposed by Cook and Campbell (1979). They describe four criteria a claim must meet before being accepted as a general law or a law applying to a practical situation. The first criterion ("statistical conclusion validity") is demonstrating statistical significance. The second and third criteria ("internal validity" and "construct validity") are demonstrating a lack of confounds. As noted, satisfying these criteria leads to the finding, which is a claim about only the subjects in the experiment.

The fourth criterion ("external validity") is demonstrating the appropriate generalization. The need for generalization at this point suggests that statistical testing does not achieve generalization; the terminology again suggests that prior to this step, the claim applies only to the subjects in the experiment.

\section{ESTABLISHING GENERALITY}

Psychologists must make claims that go beyond the subjects tested in their experiment. How do psychologists do this, and what is the role of statistical testing in this process?

\section{Generalizing to a New Population}

Cook and Campbell (1979) were concerned with the practical-application experiment. It is common in this type of experiment to test one population (e.g., college students) and to want to generalize to another population (e.g., high school students, jurors). In this case, the inferential problem is generalizing to a new population. Statistical testing does not address this problem. If the goal was to generalize to high school students, it would make little or no difference if the observed effect was known to apply to just a sample of college students or to all college students.

\section{Random Sampling}

Sometimes the goal is to make a claim about a population. For example, a pollster might wish to make a claim about what percentage of the U.S. population supports a given action by the president. With random sampling, statistics can be used to generalize to the population. Thus, random sampling from a population provides a method of generalization.

Of course, when random sampling does not occur, it does not support generalization. Therefore, this method of generalization is not used often in psychology. But suppose random sampling from a population did occur. For example, suppose an experiment compared the effectiveness of two different teaching methods using ran- dom sampling from the real population. Suppose also that Method A was observed to be more effective than Method B, and that the difference was statistically significant. With the population-based interpretation, statistical significance implies that Method A will be more effective than Method B in the population.

However, statistical significance would not imply that this finding applies to females, because an overall effect for a mixture of males and females could be caused by the effect occurring just for males. For exactly the same reason, statistical significance would not imply that this finding applies to males. If the effectiveness of Method A does not necessarily apply to either males or females, to whom would it apply? Stated paradoxically, the finding applies to everyone (the population) but not to anyone in particular.

If the experimenter recorded whether the subjects were males or female, statistical testing could support Method A being better for both male and females. However, this would not have avoided this paradox because it arises for any variable not specifically examined. Thus, with an unbiased sample from a population, statistical testing supports generalizing the observed outcome to that overall population. However, statistics does not support generalizing that outcome to any individual or subgroup in the population.

This is not a problem when the goal is to make a claim about the overall population. Pollsters trying to predict the results of an election are concerned with overall behavior, so they can be perfectly satisfied with a random sample from the population. Psychology, however, seems to be interested in the people in the population, not the overall average of the population. Or, as Sohn (1992) argued, the laws in psychology are about processes, not the parameters of real populations.

Which is to say, psychology does not seem to be that interested in the generalization that would be provided by random sampling from a population. Suppose it was already known that one teaching method worked better for one type of student and a second teaching method worked better for another type of student. I do not think psychology as a science would have much interest in knowing which of these two methods was better overall.

\section{Using the Process-Based Interpretation}

With the process-based interpretation, statistical testing does not provide generality-it is used to establish the finding, which applies to only the particular subjects tested. This is no more general than the observed result. However, this finding can be used to support more general claims.

Induction. The pattern of simple induction proceeds from instances to a general law. For example, after observing a number of green plums that are sour, one might use induction to form the general claim that all green plums are sour. For the present purposes, an important feature of induction is that the individual instances are in the same 
form as the general law. Therefore, support for a law such as "frustration increases aggression" would require individual instantiations of frustration increasing aggression.

A single observation of a frustrated person displaying aggression would not suffice. The problem is, the person might have displayed aggression even if not frustrated. Put another way, the claim that frustration increased aggression is causal. As Hume noted, causality cannot be observed. Instead, all that can be observed is aggression following frustration.

Fortunately, statistical testing provides the jump from noncausal observation to a causal claim. The finding of an experiment could be that, for the subjects tested, frustration increased aggression. This would be an instantiation and hence support for the general claim that frustration increases aggression. Thus, the finding can be used to support a general law via induction.

In actual psychology, if the same finding was established in a variety of different situations, psychologists would have confidence in the general law associated with those findings. If a finding occurred in some situations and did not occur in others, psychologists would attempt to formulate laws identifying the situations in which the effect holds and those in which it does not.

Confirmation and disconfirmation. As Mook (1983) noted, theories and laws make predictions about what will happen in an experiment. When these predictions are correct, the theory or law is confirmed; when they are wrong, the theory or law is disconfirmed. Thus, the finding of an experiment, which applies only to the subjects in the experiment, can be used to test a general law or theory applying to a large population.

For example, a study examining well-educated Boston parents showed that they rewarded their children for saying sentences that were true, not sentences that were grammatical (Brown \& Hanlon, 1970). This finding, by itself and without any generalization to other cities or socioeconomic classes, disconfirms the general law that all parents reward their children for saying sentences that are grammatical. It also suggests that a reinforcement theory of grammar learning is not correct.

Thus, experimenters do not need statistical testing to provide generalization to a larger population. Instead, even though the finding itself is not general, it can be used to support general laws and theories.

\section{MISCONCEPTIONS GENERATED BY THE ASSUMPTION OF RANDOM SAMPLING}

The population-based interpretation, being both wrong and yet used formally as the fundamental underpinning of statistical testing, has generated several misconceptions.

\section{When the Whole Population Is Sampled}

One misconception is that statistical testing would be unnecessary if the whole population were sampled (e.g., Bakan, 1966; Morrison \& Henkel, 1969). When the goal is to make a claim about how scores were produced, statistical testing is still needed to address the possibility of an observed pattern in the data being caused just by chance fluctuation.

Imagine the planet of Forty, which has only 40 inhabitants who never die. The psychologists always have 40 subjects in their experiments. The psychologists do not care about generalizing their results to other Fortians, because there are no other Fortians. (In this example, the psychologists cannot even conceptualize the possible existence of other Fortians.) An experimenter wants to know which method is best for teaching reading to Fortians, Method A or Method B. Fortunately, 20 of the Fortians learned with Method A and 20 learned with Method B, with method of learning being randomly assigned, so the experimenter measures the reading speed of each Fortian. (Assume for the sake of this example that the measurement of reading speed is perfectly accurate and reliable.) The average reading speed for Fortians who learned with Method A is faster than that of Fortians learning with Method B.

Statistical testing is needed, despite complete knowledge of the population. The Fortian experimenter wants to know if Method A is better than Method B, not whether the population of people learning with Method A is better than the population of people learning with Method B. The first issue is whether this difference could have been caused by chance, which is addressed with statistical testing.

The Fortian example is imaginary, but a possible reallife analogue would be a study of all the remaining speakers of a dying language or a study of all of the split-brain patients in the world. Or suppose a teacher is administering a test to his/her class and notices that two students sitting together seem to be cheating. The teacher then finds that these two students have the same answers for all questions. The teacher could construct a value of $p$ for the two tests being exactly the same if the two students were answering independently. There is no population for the teacher to generalize to; instead, the teacher's goal is to make inferences about the process by which these two students' answers were produced.

\section{Sampling With Replacement}

For scores to be identically distributed, they must be randomly sampled from the same population. Therefore, when the population is finite, sampling must occur with replacement (see, e.g., Hahn \& Meeker, 1991). In practice, experimenters sample without replacement.

The process-based interpretation is consistent with the practice of experimenters. The process has some mean $\mu$. Producing scores via that process (i.e., testing a subject) does not influence the process. Therefore, there is no need for "replacement." On the other hand, if a subject is tested twice, the two processes were not independent; it is inappropriate to test a subject twice and then knowingly treat these two scores as independent.

\section{Justifying Random Assignment}

When subjects are assigned randomly to groups, the groups will vary on almost every possible subject variable. 
Why are these differences between groups not confounds, posing alternative explanations? In other words, given that random assignment creates differences between groups, why is it an acceptable method of control? It is difficult to find a textbook in research methodology that provides the correct explanation. This common misconception concerning the justification of random assignment might also be a consequence of the assumption of random sampling.

The commonly offered justification is that with enough subjects assigned to each group, the groups' averages will tend to even out on all variables (see, e.g., Cook \& Campbell, 1979 , p. 5, pp. 341-342). If this justification were valid, it would still be weak, because it (1) does not justify random assignment for a small number of subjects and (2) does not explain why the small differences for large numbers of subjects is not a problem.

In fact, this justification is invalid. With more subjects, a smaller difference between groups is needed for statistical significance. The size of difference needed for statistical significance shrinks at exactly the same rate as the expected difference between groups produced by random assignment. Therefore, the efficacy of random assignment to even out the groups is independent of the number of subjects.

There are a variety of ways of expressing the proper justification of random assignment. Fisher (1949) put it this way: If there were no differences between subjects (e.g., imagine a physicist studying electrons), the error term in a statistical model would reflect only measurement error. When there are differences between subjects, with random assignment these differences can be included in the error term of the model. Any difference between groups could be caused by error-measurement error or fluctuations due to random assignment. Statistical testing addresses both of these possibilities simultaneously because it addresses whether "error" could have produced the observed difference between groups.

Reframing this explanation in terms of a processbased interpretation, statistical significance allows the conclusion that a difference between groups was not caused by chance fluctuation. In doing so, it addresses both the abstract possibility that the difference between groups was due to chance and the concrete possibility that the difference between groups was caused by chance fluctuations in random assignment or measurement error.

Thus, the process-based interpretation easily accommodates this justification. The population-based interpretation does not, perhaps because it addresses the role of chance fluctuation in sampling, not in the assignment of subjects. In that interpretation, the null hypothesis could be stated as being that the two samples were drawn from the same population. Rejecting this null hypothesis implies that the samples were drawn from different populations with different means. The question would then be, what difference between the two populations caused the difference in the independent variable? One answer would seem to be the differences between the populations caused by random assignment. This would be particularly plausible if the populations were constructed post hoc to match the samples-as, for example, with a hypothetical population.

This inferential failure seems appropriate. Suppose random samples are taken from people who drink coffee and people who do not, and a statistically significant difference is observed in number of heart attacks. With the population-based interpretation, rejecting the null hypothesis would imply that the two samples had been drawn from populations with different means. At this point, it would not be appropriate to conclude that the difference in heart attacks was caused by drinking coffee.

\section{Statistical Versus Substantive Hypotheses}

A distinction is sometimes made between the "statistical" hypothesis and a "substantive" (or "research") hypothesis. One reason for the need for two different hypotheses is that with the population-based interpretation, the statistical hypothesis is about populations, whereas the substantive hypothesis is almost always about process. For example, Keppel (1991) wrote, "Typically, but not always, a research hypothesis asserts that the treatments will produce an effect. .. . Statistical hypotheses consist of a set of precise hypotheses about the parameters of the different treatment populations" (p. 25).

In the process-based interpretation, the statistical hypothesis can also be about process. Using a process-based interpretation might not eliminate the need for two hypotheses. The research hypothesis is likely to be stated in terms that assume a lack of confounds, whereas the statistical hypothesis would include the possibility of confounds. However, the two will be closer in form with the process-based interpretation, and the inferential process connecting the two will be obvious.

\section{CONCLUSION}

The notion of random sampling from a population has not served psychology well. First, it does not justify treating scores as iids, because it rarely (if ever) occurs. Offering it as justification invites the impression that psychological experiments are methodologically flawed, which cannot be good for psychology's public image. Second, it leads to the conclusion that the goal in statistical testing is to establish the parameters of a population and that generality in psychology is achieved through statistical testing. I have argued that this is not true. Instead, in psychology the goal of statistical testing is almost always to establish a claim about process, such as that an observed difference between groups should not be attributed to chance fluctuation. Statistical significance, coupled with a lack of confounds, then leads to the finding, which in the true experiment is a causal claim about the subjects tested in the experiment. This finding is then used to support laws and theories with generality.

It is easy to justify the assumption that subjects' scores were produced by some process. The process-based interpretation accurately describes the outcome of statistical 
testing. It supplies an understanding of statistical testing that fits the practice of experimenters. It explains why random assignment is a valid method of control and why subjects should not participate in the same experiment twice. It allows the statistical hypothesis to better resemble the research hypothesis, and it clears the way for a better analysis of how psychology achieves generality.

\section{REFERENCES}

BAKAN, D. (1966). The test of significance in psychological research. Psychological Bulletin, 66, 423-437.

BRADBURY, I. (1987). Analysis of variance versus randomization testsA comparison. British Journal of Mathematical \& Statistical Psychology, 40, 177-187.

Brown, R., \& HANLON, C. (1970). Derivational complexity and order of acquisition in child speech. In J. R. Hayes (Ed.), Cognition and the development of language (pp. 1-53). New York: Wiley.

CoOK, T. D., \& CAMPBELL, D. T. (1979). Quasi-experimentation: Design and analysis issues for field settings. Chicago: Rand McNally.

EDGINGTON, E. S. (1966). Statistical inference and nonrandom samples. Psychological Bulletin, 66, 485-487.

Edgington, E. S. (1987). Randomization tests (2nd ed.). New York: Dekker.

FISHER, R. A. (1949). The design of experiments (5th ed.). Edinburgh: Oliver \& Boyd.

FRICK, R. W. (1996). The appropriate use of null hypothesis testing. Psychological Methods, 1, 379-390.

GIERE, R. N. (1973). Review of Mellor's The matter of chance. Ratio, $15,149-155$.

GoDambe, V. P. (1955). A unified theory of sampling from finite populations. Journal of the Royal Statistical Society: Series B, 17, 268-278.

HAHN, G. J., \& MEEKER, W. Q. (1991). Statistical intervals: A guide for practitioners. New York: Wiley.

KENDALL, M. G. (1952). The advanced theory of statistics. New York: Hafner.

KePPEL, G. (1991). Design and analysis (3rd ed.). Englewood Cliffs, NJ: Prentice-Hall.

Martin-Lof, P. (1969). The literature on von Mises kollectivs revisted. Theoria, 35, 12-37.

Моoк, D. G. (1983). In defense of external validity. American Psychologist, 38, 379-387.

Morrison, D. E., \& HENkel, R. E. (1969). Significance tests reconsidered. American Sociologist, 4, 131-140.

POPPER, K. R. (1990). A world of propensities. Bristol, U.K.: Thoemmes.

SoHN, D. (1992). Knowledge in psychological science: That of process or of population? Journal of Psychology, 126, 5-16.

STUDENT [GosSET, W.S.] (1908). The probable error of a mean. Biometrika, 6, 1-25.

VON MISES, R. (1957). Probability, statistics, and truth. New York: Macmillan. (Original work published 1951)

WALlis, W. A., \& RoBerTs, H. V. (1956). Statistics: A new approach. New York: Free Press.

\section{NOTE}

1. Unlike the assumption of random sampling, the assumption of independence is taken seriously. When it is violated, either the statistical test takes dependence into account, or the test is not valid. For example, multiple measurements from the same subject are not independent and would not be appropriately treated as independent.

\section{APPENDIX}

In this article, it is claimed that subjects' scores can be interpreted as random variables on the basis of the process by which they were produced. Justifying that claim requires a consideration of the meaning of probability.

\section{Physical Versus Epistemic Probabilities}

Assume a coin is going to be tossed to settle a bet. Just prior to the coin toss, the two bettors are informed that the coin is biased, but they are not told the direction of the bias.

At this point, two different types of probabilities can be distinguished. Epistemic probabilities (also called "subjective probabilities") are based on knowledge. The bettors have exactly the same information about heads versus tails, and hence no reason to prefer one versus the other. Therefore, the epistemic probability of heads equals the epistemic probability of tails; hence both equal $1 / 2$.

The second type of probability is physical (with other names being "empirical" and "objective"). The coin toss is not a fully defined event-we do not know the strength of the throw, the speed of rotation of the coin, the wind currents in the room, and so on. If nothing changed from trial to trial, the outcome of the coin toss presumably would always be the same. However, the unspecified factors change from trial to trial. The incompletely defined event of the "coin toss" can be assigned a physical probability $p$ for the outcome of heads.

The physical probability is a property of the coin; it is independent of whatever anyone might know. Therefore, it is unchanged by anything the bettors might learn about the coin.

As Kendall (1952) noted, epistemic probabilities are unsuited to the calculations of statistical tests. Return to the two bettors. Statistical tests would assume that the probability of heads on the first two trials would be the probability of heads on the first trial multiplied by the probability of heads on the second trial. This works for the physical probabilities, but it does not work for the epistemic probabilities. A biased coin is more likely to have two outcomes that are the same (two heads or two tails) than two outcomes that are different (heads on one trial and tails on the other). Therefore, learning that the coin is biased does not change the epistemic probability of heads for either the first or second trial; these are still $1 / 2$. But it does change the epistemic probability for two consecutive heads; this probability is now greater than $1 / 4$. (How much greater depends on the suspected amount of bias.)

Therefore, epistemic probabilities and physical probabilities behave differently for multiple trials. Statistical calculations are based on the rules for physical probabilities--learning about 1 subject does not change the probabilities for the next subject. Therefore, the key issue for the present purposes is the meaning of physical probabilities.

\section{The Frequentist Definition}

Conventional statistical tests such as the $t$ test and $F$ test are usually assumed to be associated with a "frequentist" interpretation of physical probability. In this interpretation, events acquire their probability by being part of an infinite population of events. Simply put, the probability of an event is the proportion of that event's occurrence in the population. For example, saying that the physical probability of heads is $p$ means that if the coin was tossed an infinite number of times, the proportion of heads would be $p$. The population-based interpretation is a natural product of this interpretation of probability: Scores are justified as being random variables by being part of a population.

However, this simple definition is inadequate because the proportion of heads can converge on $1 / 2$ without the probability 
of heads being $1 / 2$. Suppose that for a coin the probability of heads is $3 / 4$ for the first trial, $5 / 8$ for the second trial, $9 / 16$ for the third trial, and in general $\left(2^{* *}(n-1)+1\right) /\left(2^{* *} n\right)$ for the $n$th trial. The proportion of heads converges on $1 / 2$, but the probability of heads on any given trial is greater than $1 / 2$. Similarly, consider the coin that has a .9 probability of heads on odd-numbered trials and a .1 probability of heads on even trials. The proportion of heads for this coin also converges on $1 / 2$, even though again the probability of heads on any given trial is never $1 / 2$. Thus, contrary to the standard frequentist definition, the probability of heads on a given trial cannot be defined by simply knowing the proportion of heads to which an infinite number of trials converges.

Von Mises (1957, pp. 24-25) suggested that the limiting value "must remain the same in all partial sequences which may be selected from the original one in an arbitrary way." This definition rules out the second counterexample - the partial sequence of odd trials, for example, converges on .9 proportion of heads. However, it does not rule out the first counterexample, in which all partial sequences also converge on $1 / 2$ (Martin-Lof, 1969). Therefore, there seems to be no valid definition of probability based on frequencies.

The simple definition will work with the additional assumption that the coin tosses are iids. However, to say that the coin tosses are iids is to say that they all have the same probability $(p)$. This is a claim about the probability of each trial. Obviously, the definition of the probability of a trial cannot use within itself the probability of a trial.

The more fundamental problem with the frequentist definition is that in reality the probability of heads for a trial can be discussed without it being a part of an infinite sequence. For example, I discussed the situation in which the probability of heads on trial $n$ is $\left(2^{* *}(n-1)+1\right) /\left(2^{* *} n\right)$. The frequentist definition does not easily capture the notion of the probability of a single trial.

For example, Bernoulli's theorem is that if the probability of heads is $p$, the proportion of heads for repeated trials will converge on $p$. This proof assumes the probability of heads for one trial. With the frequentist definition, proving Bernoulli's theorem would be absurd because the conclusion follows easily from the definition of probability. However, this proof is not considered absurd.

\section{Propensities}

A relatively new interpretation of physical probabilities is in terms of "propensity." The exact formulation of propensities is not agreed upon; the following is a single-case interpretation (see, e.g., Giere, 1973; Popper, 1990).

In this interpretation, the probability of heads is a property of the process of producing the outcome. This includes the process of selecting the coin and how the unspecified factors vary. This propensity cannot be directly observed, so it is a hypothetical construct. However, it can be measured because in a repeated number of coin tosses, the proportion of heads will converge on the propensity. From the perspective of propensities, the frequency definition of physical probability confuses a method of measuring a construct with the construct itself.

A good model for the propensity interpretation is a machine producing random numbers following some distribution. It is easy nowadays to imagine such a machine or a computer program inside a machine creating this effect. Once the machine is constructed or the program written, it has a propensity to produce numbers following some distribution. This is a property of the machine or program and exists prior to any numbers actually being produced.

The process-based interpretation of statistical testing uses the propensity interpretation of probability. The assumption is that on some test, for example, subjects have some propensity to produce scores, which can be represented as a random variable. The event "subject producing a score" is not fully defined, allowing different possible outcomes.

How do we know that the coin's probability of heads is the same on every trial? This probability is a function of the process of producing the outcome, including the selection of the coin and the way that the unspecified factors vary. These are the same for every coin flip, so the probability must be the same. Similarly, the random variable corresponding to the subject's score is also a function of the process of selecting and testing the subject, including the unspecified factors. As long as these do not change, the distribution is the same for each subject.

(Manuscript received July 18, 1996; revision accepted for publication February 26, 1997.) 